\title{
SOLUTIONS FOR STEADY PLANE ALIGNED ELECTRO-MAGNETO-GASDYNAMICS*
}

\author{
BY \\ O. P. CHANDNA (University of Windsor) \\ AND \\ N. RUDRAIAH (Bangalore University, India)
}

1. Introduction. A vast amount of research has been carried out on the motion of electrically conducting fluids, using MHD approximations, since Alfven's [1] classic work. Mathematical complexity of the phenomenon induced many researchers to adopt a rather useful alternate technique of investigating special classes of flows such as aligned or orthogonal flows. These special classes of flows in magneto-fluiddynamics yielded various solvable second order mathematical structrures. However, not much work seems to have been done in electro-magneto-fluiddynamics.

The general problem of electro-magneto-fluiddynamics is quite complex. Kingston and Power [2] gave an elegant analysis of the dynamics of two-dimensional aligned flows. This analysis establishes that if the charge density is not identically zero in a flow region, then the magnetic field must be irrotational everywhere. However, by assuming that $\vec{H}=K \rho \vec{V}$, where $K$ is some constant, for compressible flow and density is a function of pressure only, this work is restricted to flows which have irrotational acceleration vector field.

The aim of this paper is to study steady electro-magneto-gasdynamic aligned flows when the charge density is not identically zero. In this study, we take general aligned flows so that $\vec{H}=f \rho \vec{V}$, where $f$ is an arbitrary function, and we take general equation of state for the gas. This work is carried out by employing a well established fluid-dynamical technique of establishing integrability conditions for scalar fields. Ratip Berker [3], Ozoklav $[4,5]$ and Chandna [6] used this approach for ordinary gasdynamics. Chandna et al. $[7,8]$ have employed it for compressible transverse MHD flows and incompressible electro-magneto-fluid dynamic aligned flows.

The plan of this work is as follows: In Sec. 2 we recapitulate the basic equations governing the steady motion of two dimensional electro-magneto-gasdynamic aligned flows when the charge density is not identically zero. Sec. 3 contains the derivation of integrability conditions and discusses the approach for solving flow problems. In Sec. 4, solutions to four different flow examples are obtained.

\footnotetext{
${ }^{*}$ Received October 19, 1983.
} 
2. Flow equations. The steady inviscid three-dimensional flow of electro-magentogasdynamics is governed by the system [9]:

$$
\begin{gathered}
\operatorname{div}(\rho \vec{V})=0, \\
\rho(\vec{V} \text {.grad }) \vec{V}=-\operatorname{grad} p+\mu \vec{J} \times \vec{H}+q \vec{E}, \\
\operatorname{curl} \vec{H}=\vec{J}, \\
\operatorname{curl} \vec{E}=\overrightarrow{0}, \\
\operatorname{div} \vec{E}=q / \varepsilon, \\
\vec{J}=\vec{I}+q \vec{V}=\sigma[\vec{E}+\mu \vec{V} \times \vec{H}]+q \vec{V},
\end{gathered}
$$

along with the energy balance equation and an equation of state $p=p(\rho, s)$ for the gas. This is a system of sixteen fundamental equations in sixteen unknown functions $\vec{V}$ (the velocity field), $\vec{H}$ (the magnetic field), $\vec{E}$ (the electric field), $\vec{J}$ (the current density field), $q$ (the charge density function) and three thermodynamic variables $p, \rho$ and $s$. In this system of equations $\sigma, \mu$ and $\varepsilon$ are respectively the constant electrical conductivity, the constant magentic permeability and the constant permitivity of the fluid. The magnetic field $\vec{H}$ satisfies an additional equation

$$
\operatorname{div} \vec{H}=0
$$

expressing the absence of magnetic poles in flow. From Eqs. (3) and (6), we obtain

$$
\vec{I}=\vec{\nabla} \times \vec{H}-q \vec{V}, \quad \vec{E}=[\vec{\nabla} \times \vec{H}-q \vec{V}] / \sigma-\mu \vec{V} \times \vec{H} .
$$

Eliminating $\vec{E}, \vec{J}$ from Eqs. (2), (4) and (5), by using Eqs. (3) and (8), we get

$$
\begin{gathered}
\rho(\vec{V} . \operatorname{grad}) \vec{V}=-\operatorname{grad} p+\mu(\operatorname{curl} \vec{H}) \times \vec{H}+q / \sigma[\operatorname{curl} \vec{H}-q \vec{V}]-\mu q \vec{V} \times \vec{H}, \\
\operatorname{curl}[\operatorname{curl} \vec{H}-q \vec{V}-\mu \sigma \vec{V} \times \vec{H}]=\overrightarrow{0}, \\
\operatorname{div}[q \vec{V}+\mu \sigma \vec{V} \times \vec{H}]+\frac{\sigma q}{\varepsilon}=0 .
\end{gathered}
$$

Plane Flows: We now consider the flow to be two-dimensional, so that $\vec{V}$ and $\vec{H}$ lie in a plane defined by the rectangular coordinates $x, y$ and all the flow variables are functions of $x, y$.

Decomposition of the vector equations (9) and (10), into their vector components in the flow plane and their vector components perpendicular to the flow plane, yields the following four equations:

$$
\begin{gathered}
\rho(\vec{V} . \operatorname{grad}) \vec{V}=-\operatorname{grad} p+\mu(\operatorname{curl} \vec{H}) \times \vec{H}-\frac{q^{2}}{\sigma} \vec{V}, \\
q[\operatorname{curl} \vec{H}-\mu \sigma \vec{V} \times \vec{H}]=\overrightarrow{0}, \\
\operatorname{curl}[\operatorname{curl} \vec{H}-\mu \sigma \vec{V} \times \vec{H}]=\overrightarrow{0}, \\
\operatorname{curl}(q \vec{V})=\overrightarrow{0},
\end{gathered}
$$

where Eqs. (12), (13) are equivalent to (9) and Eqs. (14), (15) result from (10). 
Equation (11), for the assumed two-dimensional flow, reduces to

$$
\operatorname{div}(q \vec{V})+\sigma q / \varepsilon=0
$$

From Eq. (13) and the above analysis, we have:

THEOREM I. If the charge density $q(x, y)$ is a non-zero function in a steady plane electro-magneto-gasdynamic flow having the magnetic field $\vec{H}$ in the flow plane, then the flow is governed by the system:

$$
\begin{gathered}
\operatorname{div}(\rho \vec{V})=0, \\
\rho(\vec{V} . \operatorname{grad}) \vec{V}=-\operatorname{grad} p+\mu(\operatorname{curl} \vec{H}) \times \vec{H}-\frac{q^{2}}{\sigma} \vec{V}, \\
\operatorname{div}(q \vec{V})+\sigma q / \varepsilon=0 \\
\operatorname{curl}(q \vec{V})=\overrightarrow{0}, \\
\operatorname{curl} \vec{H}=\mu \sigma(\vec{V} \times \vec{H}), \\
\operatorname{div} \vec{H}=0
\end{gathered}
$$

of seven scalar equations in seven unknown functions $\rho(x, y), p(x, y), q(x, y), \vec{V}=$ $(u(x, y), v(x, y))$ and $\vec{H}=\left(H_{1}(x, y), H_{2}(x, y)\right)$.

Given a solution of this system, $\vec{J}, \vec{I}$ (the conduction current density) and $\vec{E}$ are obtained from

$$
\vec{J}=\vec{I}+q \vec{V}=\operatorname{curl} \vec{H}, \quad \vec{E}=-q \vec{V} / \sigma .
$$

The above system has also been obtained by Kingston and Power [2]. We study the above flows in the following sections when $\vec{V}$ and $\vec{H}$ are everywhere parallel. For such flows, Eqs. (12) and (17) are replaced by

$$
\begin{gathered}
\rho(\vec{V} \cdot \operatorname{grad}) \vec{V}=-\operatorname{grad} p-q^{2} \vec{V} / \sigma, \\
\operatorname{curl} \vec{H}=\overrightarrow{0} .
\end{gathered}
$$

3. Integrability conditions. From the definition of aligned flows, we have

$$
\vec{H}=f(x, y) \rho(x, y) \vec{V}
$$

where $f(x, y)$ is some arbitrary scalar function.

Employing (21) in Eqs. (7), (20) and using (1), we have

$$
\begin{aligned}
\vec{V} \cdot \operatorname{grad} f & =0, \\
\vec{V} \cdot \operatorname{grad}(\ln f \rho)+\operatorname{div} \vec{V} & =0, \\
\operatorname{grad}(\ln f \rho) \times \vec{V}+\operatorname{curl} \vec{V} & =\overrightarrow{0},
\end{aligned}
$$

Taking the vector product of last equation with $\vec{V}$ and using the second equation in (22) to eliminate $\vec{V} \cdot \operatorname{grad}(\ln f \rho)$, we obtain

$$
\operatorname{grad}(\ln f \rho)=\frac{(\operatorname{curl} \vec{V}) \times \vec{V}-(\operatorname{div} \vec{V}) \vec{V}}{\vec{V} \cdot \vec{V}}, \quad \vec{V} \cdot \vec{V} \neq 0 .
$$


Taking curl of Eq. (23), we get

$$
\operatorname{curl}\left[\frac{(\operatorname{curl} \vec{V}) \times \vec{V}-(\operatorname{div} \vec{V}) \vec{V}}{\vec{V} \cdot \vec{V}}\right]=\overrightarrow{0}
$$

which is the integrability condition for the function $f \rho$. This is the first integrability condition. The second one is the integrability condition for the function $q$ and we obtain it from Eqs. (15) and (16). These equations can be rewritten as

$$
\begin{gathered}
\operatorname{grad}(\ln q) \cdot \vec{V}+(\operatorname{div} \vec{V}+\sigma / \varepsilon)=0, \\
\operatorname{grad}(\ln q) \times \vec{V}+\operatorname{curl} \vec{V}=\overrightarrow{0}
\end{gathered}
$$

Taking the vector product of the second equation with $\vec{V}$ and using the first, we have

$$
\operatorname{grad}(\ln q)=\left[\frac{(\operatorname{curl} \vec{V}) \times \vec{V}-(\operatorname{div} \vec{V}+\sigma / \varepsilon) \vec{V}}{\vec{V} \cdot \vec{V}}\right] .
$$

Taking curl of (25), we get

$$
\operatorname{curl}\left[\frac{(\operatorname{curl} \vec{V}) \times \vec{V}-(\operatorname{div} \vec{V}+\sigma / \varepsilon) \vec{V}}{\vec{V} \cdot \vec{V}}\right]=\overrightarrow{0} .
$$

No integrability condition, in $\vec{V}$ alone, can be obtained for the pressure function without taking additional flow assumptions. Kingston and Power [2], in their study of compressible case, had two additional assumptions. Their study was restricted to compressible fluids having density as a function of pressure alone. Furthermore, $\vec{H}$ was taken to be directly proportional to $\rho \vec{V}$ in the flow region so that $f(x, y)$ is constant throughout. These two additional conditions, if taken in (12), (20), give

$$
\begin{aligned}
(\vec{V} \cdot \operatorname{grad}) \vec{V}= & -(\operatorname{grad} p) / \rho(p)-q^{2} \vec{V} / \sigma \rho, \\
& \operatorname{curl}(\rho \vec{V})=\overrightarrow{0} .
\end{aligned}
$$

Taking curl of the first equation and using the second equation and Eq. (15), the integrability condition for some function of pressure for such flows reads

$$
\operatorname{curl}[(\operatorname{curl} \vec{V}) \times \vec{V}]=\overrightarrow{0} \text {. }
$$

For the flows, without the above mentioned additional assumptions, we proceed as follows:

Subtracting Eq. (24) from (26), we get

$$
\operatorname{curl}[\vec{V} / \vec{V} \cdot \vec{V}]=\overrightarrow{0}
$$

This equation, in Cartesian and polar coordinates, is equivalent to

$$
\frac{\partial}{\partial x}\left(\frac{v}{u^{2}+v^{2}}\right)-\frac{\partial}{\partial y}\left(\frac{u}{u^{2}+v^{2}}\right)=0 ; \quad \frac{\partial}{\partial r}\left(\frac{r V_{2}}{V_{1}^{2}+V_{2}^{2}}\right)-\frac{\partial}{\partial \theta}\left(\frac{V_{1}}{V_{1}^{2}+V_{2}^{2}}\right)=0(27)
$$

where $u, v$ are taken to be the Cartesian components of $\vec{V}$ and $V_{1}, V_{2}$ are the polar components. 
Equations (27) imply that there exist functions $\phi(x, y)$ and $\phi(r, \theta)$ such that the velocity field $\vec{V}$ in the two coordinate systems is:

$$
\begin{aligned}
& \vec{V}=\left(\frac{\partial \phi / \partial x}{(\partial \phi / \partial x)^{2}+(\partial \phi / \partial y)^{2}}, \frac{\partial \phi / \partial y}{(\partial \phi / \partial x)^{2}+(\partial \phi / \partial y)^{2}}\right), \\
& \vec{V}=\left(\frac{r^{2}(\partial \phi / \partial r)}{r^{2}(\partial \phi / \partial r)^{2}+(\partial \phi / \partial \theta)^{2}}, \frac{r(\partial \phi / \partial \theta)}{r^{2}(\partial \phi / \partial r)^{2}+(\partial \phi / \partial \theta)^{2}}\right) .
\end{aligned}
$$

Given any $\phi(x, y)$, and therefore the velocity field, satisfying the integrability conditions (24) and (26), the charge density function $q(x, y)$ and the product function $\rho(x, y) f(x, y)$ are determined by integrating Eqs. (25) and (23). Knowing that $f(x, y)$ is constant on each individual streamline and the curves $\phi(x, y)=$ constant are orthogonal to the family of flow streamlines, we obtain the general solution for $f(x, y)$ involving an arbitrary function. Finally, employing the solutions for $\vec{V}, q$ and $\rho$ in Eq. (19), we employ the integrability condition for $p(x, y)$ to obtain the restrictions on arbitrary functions and also integrate (19) to obtain the pressure function.

4. Solutions. In this section, we investigate the solutions for four flow problems.

EXAMPLE 1. Letting

$$
\phi(r, \theta)=F(\theta), \quad F^{\prime}(\theta) \neq 0
$$

in (28), where $F$ is some arbitrary function, we have

$$
\vec{V}=r \vec{e}_{\theta} / F^{\prime}(\theta)
$$

and, therefore,

$$
\vec{\omega}=\operatorname{curl} \vec{V}=2 \vec{k} / F^{\prime}(\theta)
$$

where $\vec{e}_{r}, \vec{e}_{\theta}$ are unit vectors in the directions of $r, \theta$ increasing at any point and $\vec{k}$ is the unit vector normal to the flow plane.

Employing Eqs. (30), (31) in Eqs. (23) to (26), we find that the integrability conditions are identically satisfied and we have

$$
\begin{aligned}
\operatorname{grad}[\ln (f \rho)] & =-\frac{2}{r} \vec{e}_{r}+\frac{F^{\prime \prime}(\theta)}{r F^{\prime}(\theta)} \vec{e}_{\theta}, \\
\operatorname{grad}(\ln q) & =-\frac{2}{r} \vec{e}_{r}+\left[\frac{F^{\prime \prime}(\theta)}{r F^{\prime}(\theta)}-\frac{\sigma}{\varepsilon} \frac{F^{\prime}(\theta)}{r}\right] \vec{e}_{\theta} .
\end{aligned}
$$

Integrating these equations, we get

$$
\begin{gathered}
f(r, \theta) \rho(r, \theta)=C_{1} F^{\prime}(\theta) / r^{2}, \\
q(r, \theta)=\left(C_{2} F^{\prime}(\theta) / r^{2}\right) \exp [-\sigma F(\theta) / \varepsilon],
\end{gathered}
$$

where $C_{1}$ and $C_{2}$ are two non-zero arbitrary constants. Since $f(x, y)$ is constant on each individual streamline and $r=$ constant are the streamlines in this example, it follows that

$$
f(r, \theta)=G(r), \quad \rho(r, \theta)=C_{1} F^{\prime}(\theta) / r^{2} G(r)
$$


and

$$
\vec{H}(r, \theta)=\rho(r, \theta) f(r, \theta) \vec{V}=C_{1} \vec{e}_{\theta} / r
$$

where $G(r) \neq 0$ is an arbitrary function. Employing the above solutions for $\rho, q, \vec{v}$ and $\vec{H}$, we find that equations (1), (7), (15), (16), (20) are satisfied and Eq. (19) are

$$
\begin{gathered}
\frac{C_{1}}{r G(r) F^{\prime}(\theta)}=\frac{\partial p}{\partial r}, \\
\frac{C_{1} F^{\prime \prime}(\theta)}{G(r) F^{\prime 2}(\theta)}-\frac{C_{2}^{2} F^{\prime}(\theta) \exp [-2 \sigma F(\theta) / \varepsilon]}{\sigma r^{2}}=\frac{\partial p}{\partial \theta} .
\end{gathered}
$$

Using the integrability condition $\partial^{2} p / \partial r \partial \theta=\partial^{2} p / \partial \theta \partial r$, we find that the functions $F(\theta)$ and $G(r)$ must satisfy

$$
C_{1}\left[\frac{r^{3} \dot{G}(r)}{G^{2}(r)}-\frac{r^{2}}{G(r)}\right]\left[\frac{F^{\prime \prime}(\theta)}{F^{\prime 2}(\theta)}\right]-\frac{2 C_{2}^{2}}{\sigma} F^{\prime}(\theta) \exp \left[-\frac{2 \sigma}{\varepsilon} F(\theta)\right]=0
$$

for the flow. Here $\dot{G}(r)$ is the differentiation of $G(r)$ with respect to $r$.

Differentiating Eq. (36) with respect to $r$, we get

$$
\frac{d}{d r}\left[\left\{\frac{r^{3} \stackrel{\circ}{G}(r)}{G^{2}(r)}-\frac{r^{2}}{G(r)}\right\}\right]\left[\frac{F^{\prime \prime}(\theta)}{F^{\prime 2}(\theta)}\right]=0 .
$$

Rejecting the possibility $F^{\prime \prime}(\theta)=0$, since $C_{2} \neq 0$, we find that $G(r)$ must satisfy

$$
\dot{G}(r)-G(r) / r-C_{3} G^{2}(r) / r^{3}=0
$$

where $C_{3}$ is an arbitrary non-zero constant. General solution of this is

$$
G(r)=r^{2} /\left(C_{3}+C_{4} r\right)
$$

where $C_{4}$ is an arbitrary constant.

Using (37) in (36), we find $F(\theta)$ must satisfy the differential equation

$$
C_{1} C_{3} \sigma F^{\prime \prime}(\theta)-2 C_{2}^{2} F^{\prime 3}(\theta) \exp [-2 \sigma F(\theta) / \varepsilon]=0 .
$$

General solution of (38) is given by

$$
C_{2}^{2} \varepsilon^{2} \exp [-2 \sigma F(\theta) / \varepsilon]+2 C_{5} \sigma^{3} F(\theta)+2 C_{1} C_{3} \sigma^{3} \theta+2 C_{6} \sigma^{3}=0
$$

where $C_{5}$ and $C_{6}$ are arbitrary constants.

Using any solution $F(\theta)$ of (39) in Eq. (35) and integrating, we find that $p(r, \theta)$ is given by

$$
p(r, \theta)=C_{7}-\frac{C_{1} C_{3}}{2 r^{2}}+\frac{C_{1} C_{4}}{r} \frac{1}{F^{\prime}(\theta)}
$$

where $C_{7}$ is an arbitrary constant.

Summing up, we have

THEOREM II. If the streamlines in a steady plane electro-magneto-gasdynamic aligned flow are a family of concentric circles $r=$ constant, then the solutions are given by Eqs. (30), (33), (34), (40) wherein $G(r)$ is given by (37) and $F(\theta)$ is any solution of (39). 
Using the solutions obtained, we find the electric field and the conduction current density from (18).

EXAMPLE 2. Taking

$$
\phi(r, \theta)=F(\alpha) \mid \alpha=r \exp (-\theta), \quad F^{\prime}(\alpha) \neq 0
$$

where $F(\alpha)$ is any function, Eq. (28) yields

$$
\vec{V}=\frac{\exp (\theta)}{2 F^{\prime}(\alpha)} \vec{e}_{r}-\frac{\exp (\theta)}{2 F^{\prime}(\alpha)} \vec{e}_{\theta}
$$

and, therefore,

$$
\vec{\omega}=\operatorname{curl} \vec{V}=-\frac{\exp (\theta)}{r F^{\prime}(\alpha)} \vec{K}
$$

Here $\vec{e}_{r}, \vec{e}_{\theta}$ and $\vec{K}$ are unit vectors at any point along $r$-increasing, $\theta$-increasing and normal to the flow plane. From Eq. (42), the streamlines for our flow are the family of curves $r \exp (\theta)=$ const. Using the above expression for $\vec{V}$ in Eqs. (23) to (26), we find that the integrability conditions (24), (26) are satisfied and we have

$$
\operatorname{grad}[\ln (f \rho)]=\left(\frac{F^{\prime \prime}(\alpha)}{F^{\prime}(\alpha) \exp (\theta)}-\frac{1}{r}\right) \vec{e}_{r}-\left(\frac{F^{\prime \prime}(\alpha)}{F^{\prime}(\alpha) \exp (\theta)}+\frac{1}{r}\right) \vec{e}_{\theta}
$$

and

$$
\begin{aligned}
\operatorname{grad}[\ln q]= & \left(\frac{F^{\prime \prime}(\alpha)}{F^{\prime}(\alpha) \exp (\theta)}-\frac{1}{r}-\frac{\sigma F^{\prime}(\alpha)}{\varepsilon \exp (\theta)}\right) \vec{e}_{r} \\
& -\left(\frac{F^{\prime \prime}(\alpha)}{F^{\prime}(\alpha) \exp (\theta)}+\frac{1}{r}-\frac{\sigma F^{\prime}(\alpha)}{\varepsilon \exp (\theta)}\right) \vec{e}_{\theta} .
\end{aligned}
$$

Integration of the above equations yields

$$
f(r, \theta) \rho(r, \theta)=\frac{D_{1} F^{\prime}(\alpha)}{r \exp (\theta)}
$$

and

$$
q(r, \theta)=\frac{D_{2} F^{\prime}(\alpha)}{r \exp (\theta)} \exp \left[-\frac{\sigma}{\varepsilon} F(\alpha)\right]
$$

where $D_{1} \neq 0, D_{2} \neq 0$ are two arbitrary real constants. Using the fact that $f(r, \theta)$ is constant on each individual streamline, Eq. (44) gives

$$
\rho(r, \theta)=\frac{D_{1} F^{\prime}(\alpha)}{\beta G(\beta)}, \quad f(r, \theta)=G(\beta) ; \quad \beta=r \exp (\theta)
$$

where $G(\beta) \neq 0$ is an arbitrary function. Furthermore, using Eqs. (42) and (46) in (21), we get

$$
\vec{H}=D_{1}\left(\vec{e}-\vec{e}_{\theta}\right) / 2 r .
$$


Using the above solutions, Eqs. (1), (7), (15), (16) and (20) are satisfied. However, Eq. (19), by the use of above solutions, take the form

$$
\frac{D_{1} F^{\prime \prime}(\alpha)}{r G(\beta) F^{\prime 2}(\alpha)}+\frac{D_{1} \exp (\theta)}{r^{2} G(\beta) F^{\prime}(\alpha)}-\frac{D_{2}^{2} F^{\prime}(\alpha) \exp [-2 \sigma F(\alpha) / \varepsilon]}{\sigma r^{2} \exp (\theta)}=2 \frac{\partial p}{\partial r}
$$

and

$$
\frac{D_{2}^{2} F^{\prime}(\alpha) \exp [-2 \sigma F(\alpha) / \varepsilon]}{\sigma r \exp (\theta)}-\frac{D_{1} F^{\prime \prime}(\alpha)}{G(\beta) F^{\prime 2}(\alpha)}=2 \frac{\partial p}{\partial \theta} .
$$

Applying the integrability condition $\partial^{2} p / \partial r \partial \theta=\partial^{2} p / \partial \theta \partial r$, we find that $F(\alpha)$ and $G(\beta)$ must satisfy

$$
\begin{gathered}
D_{1}\left[\left(\frac{F^{\prime \prime}(\alpha)}{F^{\prime 2}(\alpha)}\right)\left\{\frac{1}{r G(\beta)}-\frac{2 \exp (\theta) \dot{G}(\beta)}{G^{2}(\beta)}\right\}\right. \\
\left.+\left(\frac{1}{F^{\prime}(\alpha)}\right)\left\{\frac{\exp (\theta)}{r^{2} G(\beta)}-\frac{\exp (2 \theta) \dot{G}(\beta)}{r G^{2}(\beta)}\right\}\right] \\
+\frac{2 D_{2}^{2} F^{\prime}(\alpha) \exp [-2 \sigma F(\alpha) / \varepsilon]}{\sigma r^{2} \exp (\theta)}=0
\end{gathered}
$$

where $\dot{G}(\beta)$ is the differentiation of the function $G$ with respect to its argument.

Now in the flow plane, with the exception of the point $r=0$, the variables $\alpha=r \exp (-\theta)$ and $\beta=r \exp (\theta)$ are independent variables. Expressing Eq. (49) in $\alpha, \beta$, we get

$$
\begin{aligned}
{\left[\frac{\beta}{G(\beta)}\right]\left[\frac{F^{\prime \prime}(\alpha)}{F^{\prime 2}(\alpha)}+\frac{1}{\alpha F^{\prime}(\alpha)}\right] } & -\left[\frac{\beta^{2} \dot{G}(\beta)}{G^{2}(\beta)}\right]\left[\frac{2 F^{\prime \prime}(\alpha)}{F^{\prime 2}(\alpha)}+\frac{1}{\alpha F^{\prime}(\alpha)}\right] \\
& +\frac{2 D_{2}^{2} F^{\prime}(\alpha) \exp [-2 \sigma F(\alpha) / \varepsilon]}{D_{1} \sigma}=0
\end{aligned}
$$

Differentiating (50) with respect to $\beta$, we have

$$
\begin{aligned}
& {\left[\frac{F^{\prime \prime}(\alpha)}{F^{\prime 2}(\alpha)}+\frac{1}{\alpha F^{\prime}(\alpha)}\right]\left[\frac{d}{d \beta}\left\{\frac{\beta}{G(\beta)}\right\}\right]} \\
& \quad-\left[\frac{2 F^{\prime \prime}(\alpha)}{F^{\prime 2}(\alpha)}+\frac{1}{\alpha F^{\prime}(\alpha)}\right]\left[\frac{d}{d \beta}\left\{\frac{\beta^{2} \dot{G}(\beta)}{G^{2}(\beta)}\right\}\right]=0 .
\end{aligned}
$$

If either of the differential expressions $F^{\prime \prime}(\alpha) / F^{2}(\alpha)+1 / \alpha F^{\prime}(\alpha)$ and $2 F^{\prime \prime}(\alpha) / F^{\prime 2}(\alpha)+$ $1 / \alpha F^{\prime}(\alpha)$ is identically zero in Eq. (51), then Eq. (50) is not satisfied for any set of $F(\alpha)$ and $G(\beta)$. Therefore, for the determination of functions $F(\alpha)$ and $G(\beta)$ such that Eqs. (50) and (51) are simultaneously satisfied, we have the following two cases:

(i) $d[\beta / G(\beta)] / d \beta=0$ and, therefore, $d\left[\beta^{2} \dot{G}(\beta) / G^{2}(\beta)\right] / d \beta=0$,

(ii) $d[\beta / G(\beta)] / d \beta \neq 0$.

We study the two cases. 
Case I. In this case,

$$
G(\beta)=D_{3} \beta
$$

where $D_{3}$ is a non-zero arbitrary constant.

Using (52) in (50), we find that $F(\alpha)$ satisfies the differential equation

$$
D_{1} \sigma F^{\prime \prime}(\alpha)-2 D_{2}^{2} D_{3} F^{\prime 3}(\alpha) \exp \left[-\frac{2 \sigma}{\varepsilon} F(\alpha)\right]=0 .
$$

General solution for $F(\alpha)$ satisfying this equation is given by

$$
D_{2}^{2} D_{3} \varepsilon^{2} \exp \left[-\frac{2 \sigma}{\varepsilon} F(\alpha)\right]+D_{4} F(\alpha)+2 D_{1} \sigma^{3} \alpha+D_{5}=0
$$

where $D_{4}$ and $D_{5}$ are arbitrary constants. Using (52) in (48) and transforming the resulting equations to $\alpha, \beta$ coordinates, we get

$$
\begin{aligned}
& 4 \frac{\partial p}{\partial \alpha}=\left(\frac{D_{1}}{D_{3}}\right) {\left[\frac{2 F^{\prime \prime}(\alpha)}{\alpha \beta F^{\prime 2}(\alpha)}+\frac{1}{\alpha^{2} \beta F^{\prime}(\alpha)}\right] } \\
&-\left(\frac{2 D_{2}^{2}}{\sigma}\right)\left[\frac{F^{\prime}(\alpha) \exp \left\{-\frac{2 \sigma}{\varepsilon} F(\alpha)\right\}}{\alpha \beta}\right], \\
& 4 \frac{\partial p}{\partial \beta}=\left(\frac{D_{1}}{D_{3}}\right)\left[\frac{1}{\alpha \beta^{2} F^{\prime}(\alpha)}\right] .
\end{aligned}
$$

Integrating Eq. (55), with the use of (53), we have

$$
p(r, \theta)=D_{6}-\frac{D_{1}}{4 D_{3} r^{2} F^{\prime}(\alpha)}
$$

where $D_{6}$ is an arbitrary constant.

Case II. In this case, by using separation of variables, Eq.(51) splits up into two differential equations

$$
\frac{d}{d \beta}\left[\frac{\beta^{2} \dot{G}(\beta)}{G^{2}(\beta)}\right]-L_{1} \frac{d}{d \beta}\left[\frac{\beta}{G(\beta)}\right]=0
$$

and

$$
\left(2 L_{1}-1\right) \alpha F^{\prime \prime}(\alpha)+\left(L_{1}-1\right) F^{\prime}(\alpha)=0
$$

for the functions $F(\alpha)$ and $G(\beta)$. Here $L_{1}$ is an arbitrary constant which cannot be zero, 1 or $\frac{1}{2}$. Solving these differential equations, we get

$$
G(\beta)=\frac{\left(1-L_{1}\right) \beta^{L_{1}}}{L_{2} \beta^{L_{1}-1}+L_{3}\left(1-L_{1}\right)}
$$

and

$$
F(\alpha)=\left(\frac{2 L_{1} L_{4}-L_{4}}{L_{1}}\right) \alpha^{\left.L_{1} / 2 L_{1}-1\right)}+L_{5}
$$


where $L_{2}, L_{3}, L_{4}$ and $L_{5}$ are arbitrary constants. Using these solutions in Eq. (50), we find that Eq. (50) is not satisfied. Therefore, for the flow problem, we conclude:

THEOREM III. If the streamlines in a steady plane electro-magneto-gasdynamic aligned flow are a family of spirals $r e^{\theta}=$ constant, then the solutions to the flow problem are given by the Eqs. (42), (45), (46), (47), (56) with $G(\beta)$ and $F(\alpha)$ given by Eqs. (52) and (54) respectively.

EXAMPLE 3. We let

$$
\phi(r, \theta)=F(r) ; \quad F^{\prime}(r) \neq 0
$$

and proceed, as in previous examples, to obtain

$$
\begin{aligned}
& \vec{V}=\frac{1}{F^{\prime}(r)} \vec{e}_{r}, \quad \vec{\omega}=\overrightarrow{0}, \quad \rho=\frac{A_{1} F^{\prime}(r)}{r G(\theta)}, \quad f=G(\theta), \\
& q=\frac{A_{2} F^{\prime}(r)}{r} \exp \left[-\frac{\sigma}{\varepsilon} F(r)\right], \quad \vec{H}=\frac{A_{1}}{r} \vec{e}_{r}
\end{aligned}
$$

where $A_{1}, A_{2}$ are non-zero arbitrary constants and $G(\theta)$ is a non-zero arbitrary function. The flow is a radial flow with origin as its singular point.

Using these solutions in flow equations, we find that with the exception of linear momentum equations all other equations are satisfied and the linear momentum equations read

$$
\begin{aligned}
& \frac{\partial p}{\partial r}=\frac{A_{1} F^{\prime \prime}(r)}{r F^{\prime 2}(r) G(\theta)}-\frac{A_{2}^{2} F^{\prime}(r) \exp [-2 \sigma F(r) / \varepsilon]}{\sigma r^{2}} \\
& \frac{\partial p}{\partial \theta}=0 .
\end{aligned}
$$

Using the integrability condition for $p$, we find the following possibilities:

(i) $F(r)=C_{3} r+C_{4} \mid C_{3} \neq 0, C_{4}$ are arbitrary constants and $G(\theta)$ is any function of $\theta$.

(ii) $G(\theta)=C_{5} \mid C_{5} \neq 0$ is an arbitrary constant and $F(r)$ is an arbitrary function.

In either of these cases $p=p(r)$ is given by integration of Eqs. (59). Therefore,

THEOREM IV. If the flow is a radial flow with $\theta=$ constant as its streamlines, then solutions are given by Eqs. (58) and (59).

EXAMPLE 4. Letting

$$
\phi(x, y)=F(\alpha) ; \quad \alpha=a y-b x, F^{\prime}(\alpha) \neq 0
$$

and proceeding as in previous examples, we find that

$$
\begin{aligned}
& \vec{V}=\left(\frac{-b}{\left(a^{2}+b^{2}\right) F^{\prime}(\alpha)}, \frac{a}{\left(a^{2}+b^{2}\right) F^{\prime}(\alpha)}\right), \quad \rho=\frac{B_{1} F^{\prime}(\alpha)}{G(\beta)}, \\
& q=B_{2} F^{\prime}(\alpha) \exp \left[-\frac{\sigma}{\varepsilon} F(\alpha)\right], \quad \vec{H}=\left(\frac{B_{1}}{a^{2}+b^{2}}\right)(-b, a)
\end{aligned}
$$

are the solutions to the flow problem with parallel straight streamlines $\beta=a x+b y=$ constant. Here $B_{1}, B_{2}$ are non-zero constants, $G(\beta)$ is a non-zero arbitrary function and cartesian coordinates are used. 
Using (61) in linear momentum equations in $\alpha, \beta$ coordinates, we get

$$
\left(a^{2}+b^{2}\right) \frac{\partial p}{\partial \alpha}=B_{1} \frac{F^{\prime \prime}(\alpha)}{G(\beta) F^{\prime 2}(\alpha)}-\frac{B_{2}^{2} F^{\prime}(\alpha)}{\sigma} \exp \left[-\frac{2 \sigma}{\varepsilon} F(\alpha)\right]
$$

and

$$
\frac{\partial p}{\partial \beta}=0
$$

Applying the integrability condition for $p$, we find that either $F^{\prime \prime}(\alpha)=0$ with any non-zero choice for $G(\beta)$ or $G(\beta)=$ a non-zero constant with any non-constant $F(\alpha)$. In either case, we find $p=p(a y-b x)$ by integrating Eq. (62). Therefore, we have

THEOREM V. If the flow is a straight parallel flow with $a x+b y=$ constant as its streamlines, the solutions are given by Eqs. (61) and (62).

\section{REFERENCES}

[1] H. Alfvén, Cosmical electrodynamics, Clarendon Press, Oxford, 1950

[2] J. G. Kingston and G. Power, An analysis of two-dimensional aligned-field magnetogasdynamic flows, ZAMP 19, 851-863 (1968)

[3] R. Berker, C. R. L'Acad. Sci. Paris 242, 342 (1956)

[4] H. Özoklav, J. Math. Mech. 8, 27 (1959)

[5] H. Özoklav, Arch. Ration. Mech. Analysis 15, 154 (1964)

[6] O. P. Chandna, Int. J. Engng. Sci. 9, 611 (1971)

[7]F O. P. Chandna, Canadian J. Physics, Vol. 50, 2565 (1972)

[8] O. P. Chandna, H. Toews and K. Prabaharan, Quarterly of Appl. Math., 39249 (1981)

[9] S. I. Pai, Magnetogasdynamics and plasma dynamics, Springer-Verlag, Vienna, 1962 